\title{
A Rare Case of Aicardi-Goutières Syndrome Who Showed a Positive Evolution after Being Treated with Growth Hormone, High Doses of Melatonin and Neurorehabilitation
}

Jesús Devesa 1,", Alba Alonso 2, Patricia Porto ${ }^{3}$, Ana Quintana ${ }^{4}$, María Carrillo 5, Pablo Devesa ${ }^{6}$ and Carlos I. Puell ${ }^{7}$.

${ }^{1}$ Scientific Direction. Medical Center Foltra. 15886-Teo. Spain. Email: jesus.devesa@usc.es. Phone: +34-981802928. Fax: +34-981807650.

${ }^{2}$ Children Physiotherapy. Medical Center Foltra. 15886-Teo. Spain. Email:

A.alonsogo087@gmail.com. Phone: +34-981802928. Fax: +34-981807650.

${ }^{3}$ Occupational Therapy. Medical Center Foltra. 15886-Teo. Spain. Email:

patriciaportotrillo@gmail.com Phone: +34-981802928. Fax: +34-981807650.

${ }^{4}$ Neuropsychology. Medical Center Foltra. 15886-Teo. Spain. Email:

anaquintanahernandez@gmail.com. Phone: +34-981802928. Fax: +34-981807650.

${ }^{5}$ Speech Therapy. Medical Center Foltra. 15886-Teo. Spain. Email:

marycarrillotorres@gmail.com. Phone: +34-981802928. Fax: +34-981807650.

${ }^{6}$ Research and Development. Medical Center Foltra. 15886-Teo. Spain. Email:

pdevesap@foltra.org. Phone: +34-981802928. Fax: +34-981807650.

7 Physical Medicine and Rehabilitation. Medical Center Foltra. 15886-Teo. Spain. Email: cipuell@foltra.org. Phone: +34-981802928. Fax: +34-981807650.

*Correspondence: jesus.devesa@usc.es or devesa.jesus@gmail.com; Tel.: +34-981-802-928

\begin{abstract}
1) Background: The Aicardi-Goutières syndrome (AGS) is a rare congenital disease which courses with severe psychomotor delay in neurodevelopment. We studied a 3-years and 4months old child with very important growth and weight affectation, microcephaly and loss of his developmental skills from 16-months of age, in which previous metabolic and genetic studies discarded any abnormality. Therefore diagnosis was cerebral palsy of unknown etiology. He presented spastic paraparesia, poor fine motricity, cognitive impairment and absence of oral communication. One year after discharge, a de novo mutation was detected in a single nucleotide in the gene IFIH1: c.2317G>C, being then diagnosed of AGS. 2) Methods: Blood analysis showed very low IGF-1 and slightly elevated liver transaminases. Treatment consisted in GH (0.04 $\mathrm{mg} / \mathrm{kg} /$ day), melatonin (20 mg/day, and after 3-months $50 \mathrm{mg} /$ day), and daily intense neurorehabilitation (5 days/week). Tests for evaluating childhood developmental milestones (GMFM-88, BDIST and the WeeFim test) were carried out every 3-months. 3) Results: The equivalent age at admission (10-months) increased to 24-months at discharge. There were clear improvements in spasticity, fine motor function, swallowing, cognition and autonomy as well as in communication, growth and weight. 4) Conclusion: Most likely melatonin blocked or decreased the interferon signature, allowing $\mathrm{GH}$ and neurorehabiltation to act on neurodevelopment.
\end{abstract}

Keywords: Aicardi-Goutiéres syndrome; interferon 1; melatonin; growth hormone; neurorehabilitation 


\section{Introduction}

The Aicardi-Goutières syndrome (AGS: MIM 225750) was first described in 1984 by Aicardi and Goutières, paediatric neurologists who gave their name to this neuropathology [1]. According to their description of the findings observed in eight patients, the syndrome is characterized by an early and progressive affectation of the developing brain leading to bilateral spasticity, microcephaly and a rapid evolution to brain atrophy and demyelination. They also observed calcifications in the basal ganglia and lymphocytosis in the cerebrospinal fluid (CSF). These authors postulated that the syndrome was familial and that, most likely, had a genetic origin [1]. Two years later a new case of this encephalopathy was described [2]. In this case, in addition to the findings first described by Aicardi-Goutières [1], abnormal movements of the eyes were also observed, and the child quickly evolved to a vegetative state. The authors concluded that this syndrome may be the consequence of a genetic degenerative encephalopathy, autosomal recessively transmitted [2].

A new study in a higher population of children suffering AGS, revealed that in 19 of the 27 cases studied, the clinical manifestations of the encephalopathy appeared in the first 4-months of life [3]; head circumference was normal at birth in most of these cases, but 21 of the 27 patients developed microcephaly during the first year of life. CSF lymphocytosis existed and high levels of interferon-alpha (IFN- $\alpha$ ) (or type I interferon: IFN-1) were detected both in serum and CSF in 14 of these AGS children. Moreover, IFN- $\alpha$ levels were higher in CSF than in serum, which suggested an intrathecal synthesis of this antiviral cytokine, but also that it could play a role in the pathogenesis of this encephalopathy $[3,4]$. These authors concluded that their study revealed that the syndrome was inherited in an autosomal recessive manner, a conclusion also suggested by previous and further reports [2-14]. Interestingly, studies in sibs demonstrated that AGS may course with a highly variable phenotype $[9,11,12]$, which suggests that different genes or the existence of different mutations in a single gene may be responsible for the appearance or the evolution of the AGS.

Since these pioneering studies were performed, a large number of publications reflect the heterogeneity in the type of genes involved in the onset of the syndrome and its clinical manifestations [13-17].

Here we describe the case of a child affected with an unusual presentation of AGS, who showed clear improvements after being treated with GH, high doses of Melatonin (MT) and neurorehabilitation during 16-months, in which the genetic study carried out at six years of age, 1-year after discharge, demonstrated the existence of a de novo pathogenic mutation in the IFIH1 gene (c.2317G>C).

\section{Results}

\subsection{Physiotherapy}

Initially, there was a remarkable dorsal flexion with the pelvis fixed in retroversion due to the high muscular tone in the lower limbs. The spontaneous mobility on a mat was based on turns made with great difficulty due to the global flexor pattern. He was able to maintain the prone position with the support of his hands, being able to achieve the crawling although there was an insufficient dissociation of scapular and pelvic waists. Therefore, main objectives in this therapy were: a) to potentiate the active movement in lower limbs towards a normalization of the 
muscular tone that allowed him to make movements with greater fluidity, without conditioning so much the upper part of the trunk; b) to achieve a dissociation and stability at the level of waists in order to reach a functional sedestation and better management of his upper limbs; c) straightening and extension at the trunk level that would allow him greater motor independence.

At discharge, the patient was able to maintain sedestation without plantar support and perform lateral support reactions with open hands. There were clear improvements in bipedestation, maintaining this position quite more time than at admission, improving the extension of the lower limbs. Similarly, there were improvements in bipedestation with anterior support (his hands). With regard to gait, a step with an adequate triple flexion was achieved, although the patient needed the support of the therapist for walking. Therefore, the Gross Motor Function Test [GMFM-88] increased from $17.2 \%$ at admission to $43 \%$ at discharge. These changes mean an increase from level I to level III according to the study by Hanna et al. in children with cerebral palsy [18]. Figure 1 shows the evolution in the GMFM-88 scores from admission to discharge, and Figure 2 shows the physical changes in the patient during the same period of time. Spasticity (Modified Assworth Scale [MAS]) decreased from grade 3 at admission to grade 1+ at discharge. An X-ray exam showed that there was not subluxation of hips (data not shown).

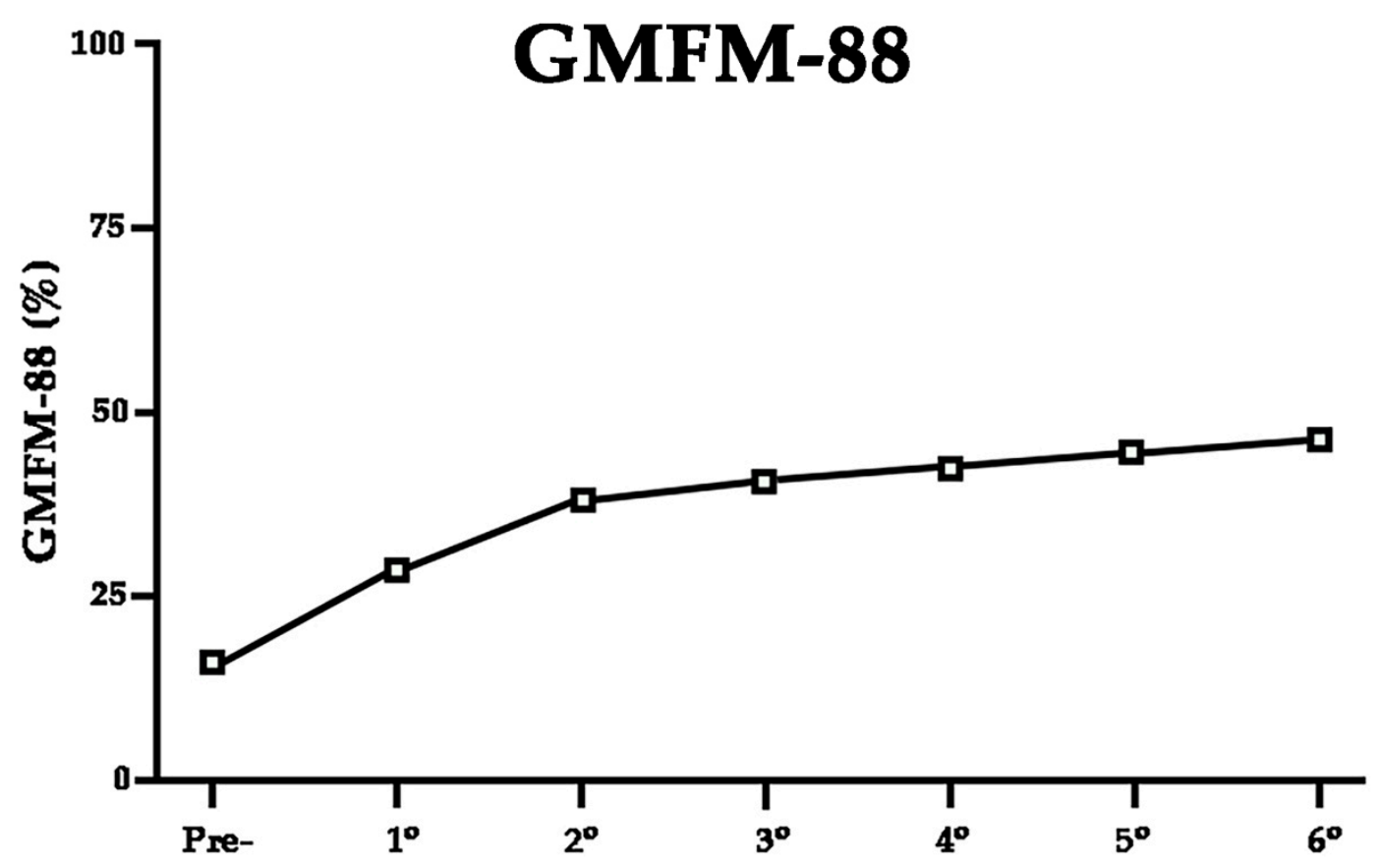

Figure 1.- Graphic representation of the scores reached in GMFM-88 from admission (Pre-) to discharge $\left(6^{\circ}\right)$. Controls were performed every 3 -months after admission. 

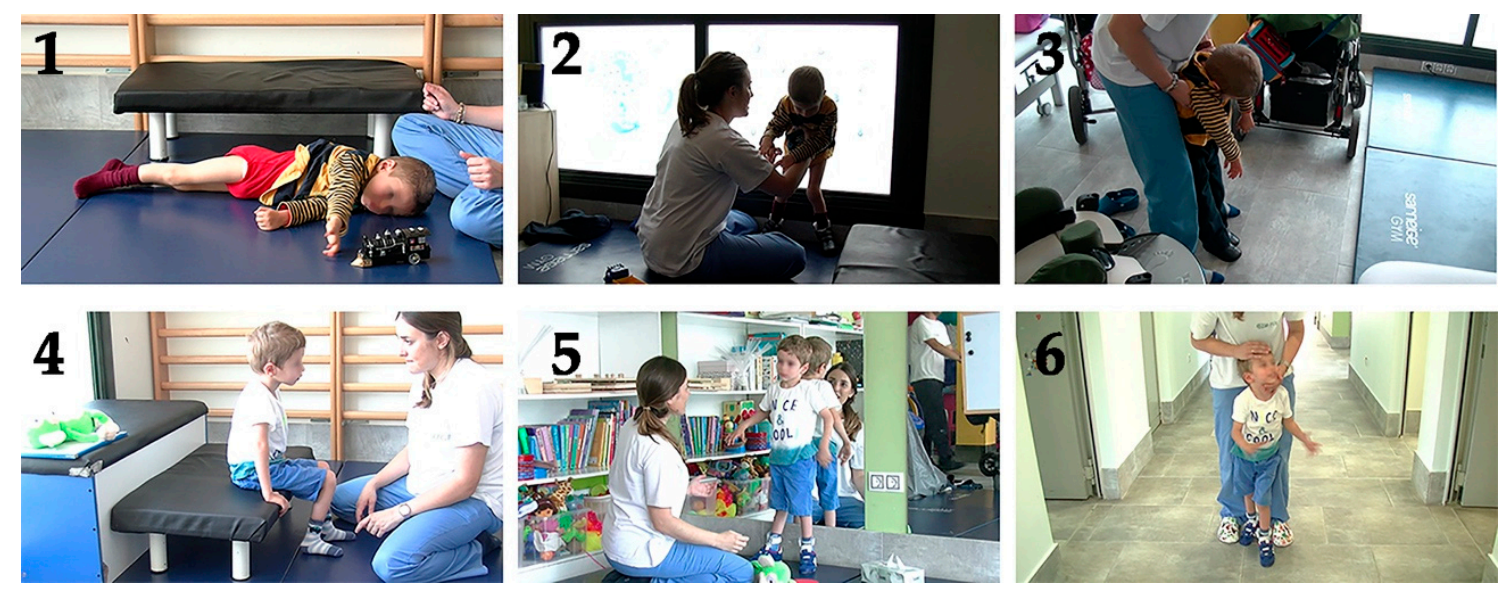

Figure 2.- Upper graph (1 to 3 ) shows the physical situation of the patient at admission. 1: Turns in mat; 2: Unstable standing, although the back is leaning against the window and the therapist is holding ankles, due to the dorsal flexion and that the pelvis is fixed in retroversion; 3: Observe dorsal flexion and impossible bipedestation. Lower graph (4 to 6) shows the situation of the patient at discharge. 4 : Note the stable sedestation and the loss of dorsal flexion; 5: Note the stable bipedestation with open hands, although the back is leaning against the glass wall; 6: Gait with support, but note the normal movement of the lower limbs.

Interestingly, one year after discharge the parents notified us that cognitive and physical evolution continued (Figure 3), and that the patient was able to move in a wheelchair autonomously. Independent gait was still imposible, mainly because of the shortening of abductors, who need a corrective surgery.

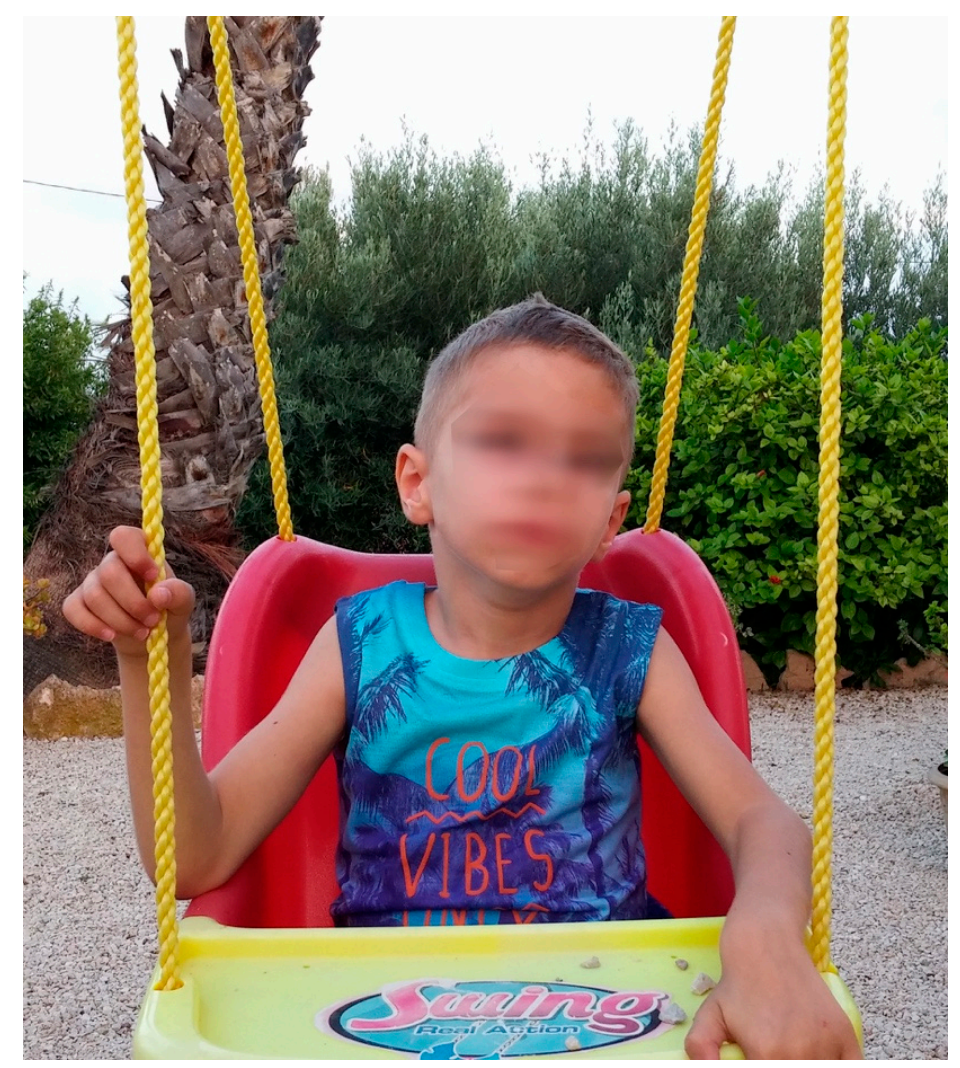

Figure 3.- One year after discharge. The patient is swinging autonomously on a swing. 


\subsection{Speech Therapy}

\subsubsection{Speech}

Before commencing the treatment the patient only was able to emit babbles, presenting disruptive behaviors with great frequency to avoid working. Progressively, improvements in the functionality and voluntary control of the orofacial musculature were observed; likewise, the patient improved the protrusion and lingual retraction movements and began to perform lateralization movements of the tongue, previously non-existent. Therefore, the patient began to be able to emit an increasing number of vocables improving intelligibility, until he began to say some words. Disruptive behaviors disappeared and at discharge there were important advances in the understanding of complex orders. Full language was not achieved, but according to reports from his parents improvements in this area continue one year after discharge.

\subsubsection{Swallowing}

Initially the patient showed a great aversion to food with marked peri and intraoral hypersensitivity, and an excessively advanced nausea reflex. Therefore, their diet was based on semi-solid food to avoid rejection and vomiting. The passage from the oral to pharyngeal phase was performed by means of suction reflex. Due to his lingual hypotonia and lingual mobility deficit there was poor management of the bolus during the oral phase of swallowing. He did not chew and did not drink by glass, he only drank by feeding bottle. Moreover, the meal times were very high. Consequently, the patient showed a clear undernourishment.

At discharge, these affectations had disappeared, and the patient was able to swallow hard solids, although not yet had gotten a complete rotational movement during mastication. His body weight increased being already in the percentile 3 (p3) for his age.

Figure 4 shows the changes observed in this area of treatment.
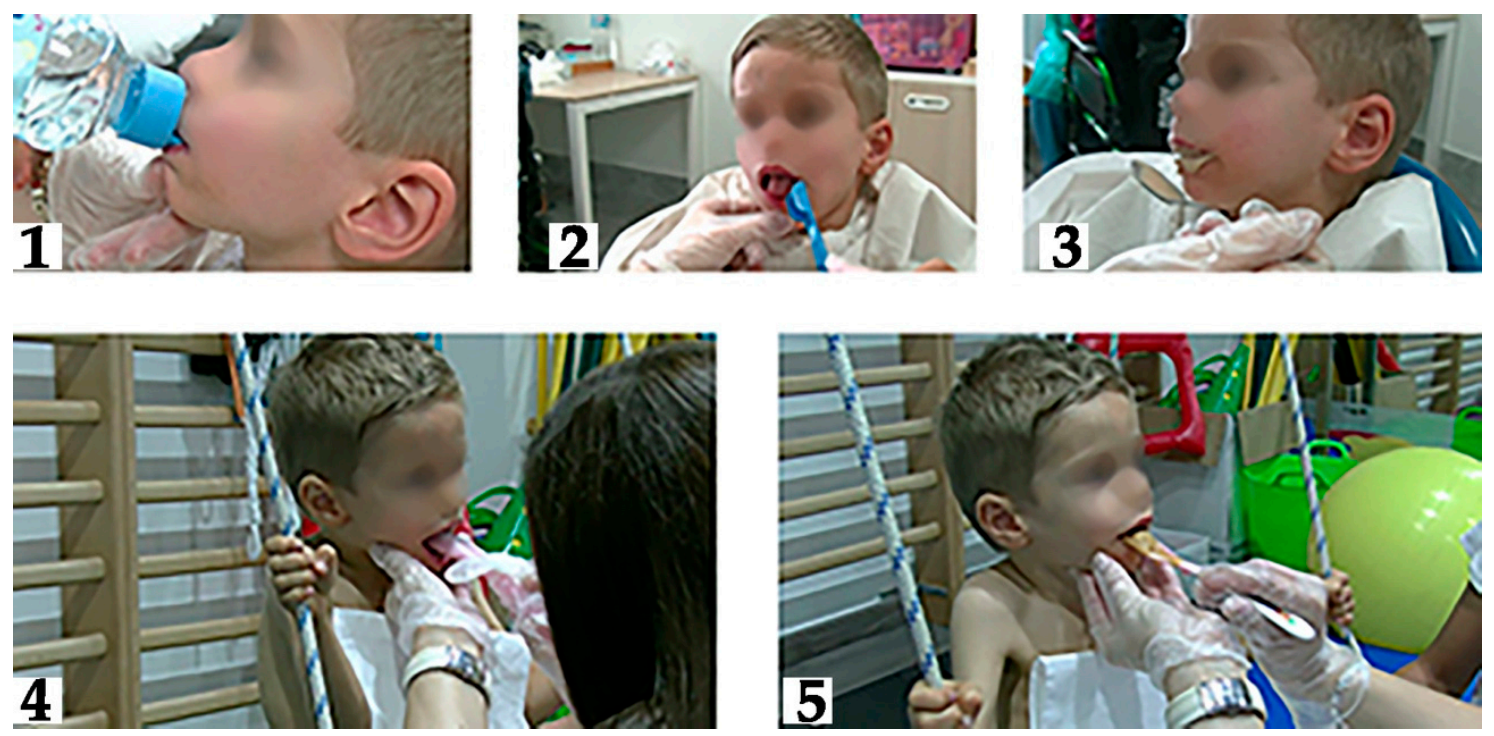

Figure 4.- Orofacial changes. Upper graph (1 to 3) shows the situation at admission. 1: The patient only drank by feeding bottle; 2: Lingual hipotony and lack of lateral movements of the tongue; 3 : Advanced nausea and vomiting reflex. Lower graph (4 and 5) shows changes at discharge. 4: The patient was able to move the tongue laterally. 5: Rejection to semi-solid food disappeared and the patient was able to swallow hard solids. 


\subsection{Neuropsychology}

At admission the patient showed a very poor social ability for interacting with other children, and he was absolutely dependent for any activity of daily life. He was not able to get his hands on the middle line. His attention span was very low, both at a sustained and selective level. Therefore, scores in the Battelle Developmental Inventory Screening Test (BDIST), which permits to evaluate the evolution of a child in five different areas (Social/personnel, Adaptive, Communication, and Cognition) were very low (Table 1, Pre-); therefore while his chronological age was 51-months, the equivalent age according to this test was 10-months. Sixteen months later, the evolution of the patient was quite positive, mainly at the cognitive level, as Table 1 (Post-) shows. He began to interact both with adults and other children, his attention improved, he began to try to find alternatives to solve single problems, he began to use the spoon or the fork to eat, and he asked for food or water. In the motor area, he was already able to make the clamp, take and deliver objects, turn pages of a book and hold the paper while drawing. His chronological age was 67-months and the equivalent age was 24-months. These data indicated that the neurodevelopment of the patient not only stopped when he was 16-months old, but also suffered a regression that began to be recovered when the patient initiated the treatment in our Medical Center, increasing his neurodevelopment in 14-months during the 16-months treatment period.

Table 1. Scores in the Battelle Developmental Inventory Screening Test at admission (Pre-) and at discharge (Post-). Note the significant improvements in all areas, excepting Gross motor functions and Expressive communication. In all, the patient increased his neurodevelopment in 14-months during the 16-months of treatment, as the difference between Total scores Post- and Pre-treatment reflects.

\begin{tabular}{lll}
\hline Area & Pre & Post \\
\hline Social/Personnel & 14 & 23 \\
Adaptive & 10 & 25 \\
Gross motor & 2 & 8 \\
Fine motor & 4 & 29 \\
Total motor & 3 & 13 \\
Receptive communication & 19 & 38 \\
Expressive communication & 14 & 19 \\
Total communication & 15 & 26 \\
Cognition & 11 & 44 \\
\hline TOTAL & $\mathbf{1 0}$ & 24 \\
\hline
\end{tabular}

\subsection{Occupational Therapy}

As in other therapies, at admission the patient showed a clear rejection to any tactile stimulation (he avoided plantar and palmar support) and showed a clear displeasure against different textures and products (creams, foams, plasticine, soft/rough or hard/soft objects), causing alterations in other areas, such as feeding). There was no sphincter control. Therefore his scores in the scale WeeFim were very low (Table 2, Pre-).

Once analyzed the patient the objectives in this therapy were: a) to favor postural control (trunk extension) and to reach active sedestation and standing, to better integrate his right upper limb and to improve manipulative dexterity and oculo-motor control; b) to favor sensory 
integration, both at the vestibular and proprioceptive levels, and reduce tactile rejection; c) to normalize eating behavior; $\mathrm{d}$ ) to achieve sphincter control.

At discharge, the patient was more independent for daily life activities. He needed lesser help for dress and undress, putting on or taking off his socks and footwear. Sphincter control had begun. Cognition improved and the patient showed a good level of understanding, memory, and problem resolution. With regard to mobility, the instrument WeeFim only detects general aspects related to it; terefore, the scores reached in this component of the scale did not change from its initial value (Table 2, Post-). However, the therapist reported clear improvements at motor level, such as better gross and fine motor, better ability for performing bimanual activities, better control and extension of the upper trumk, and better dissociation of scapular and pelvic waists, but these aspects are not examined in the WeeFim test. These motor changes are shown in Figure 5 .

Table 2.- Scores in the different areas evaluated by the WeeFim test at admission (Pre-) and at discharge (Post-). As it can be observed main changes appeared in the personal care and cognition. No changes were observed in the area examining the mobility for the reasons explained in the text.

\begin{tabular}{lll}
\hline Area & Pre & Post \\
\hline Personal care & $11 / 56$ & $25 / 56$ \\
Mobility & $5 / 35$ & $5 / 35$ \\
Cognition & $13 / 35$ & $24 / 35$ \\
\hline TOTAL & $29 / 126$ & $54 / 126$ \\
\hline
\end{tabular}
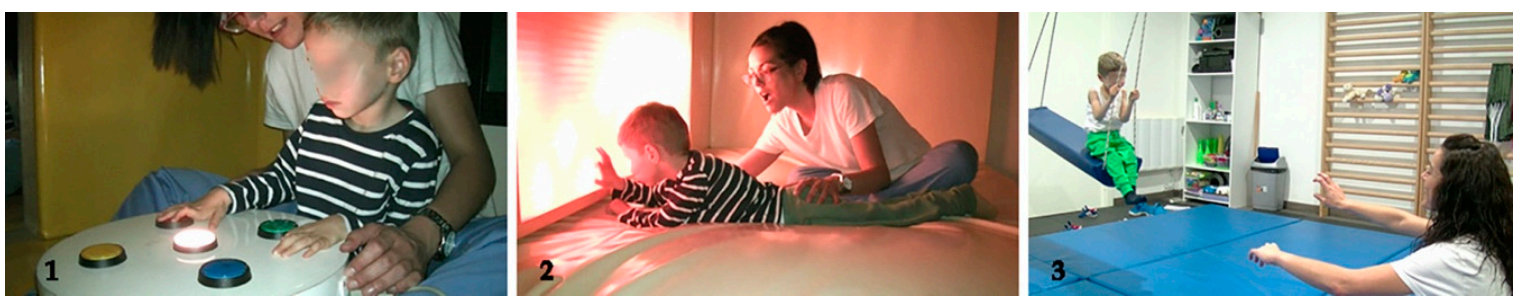

Figure 5.- Motor improvements. 1 and 2 show the patient working in the multi-sensory stimulation room Snoezelen. In both cases it can be seen how the fine motricity of the patient improved at discharge; 1 : the patient press a button to get the color required by the therapist; 2 : the patient touches the screen to change the color of it. 3: Note how sensory integration was improved, the patient balances without fear, pushed by the therapist.

\subsection{Blood Analysis}

The pre-treatment blood analysis was normal, except for a very low IGF-I value $(<25$ $\mu \mathrm{g} / \mathrm{L})$, clearly under the limits of normal values for the age of the patient $(50-300 \mu \mathrm{g} / \mathrm{L})$, while the insulin like growth factor binding protein 3 (IGFBP3) was in normal values ( $2.9 \mu \mathrm{g} / \mathrm{L}$, normal range: $0.7-3.9 \mu \mathrm{g} / \mathrm{L}$ ). Plasma liver transaminases GOT and GPT were increased to values slightly higher than normal upper range (GOT: $46 \mathrm{U} / \mathrm{L}$, range: 10-40; GPT: $42 \mathrm{U} / \mathrm{L}$, range: 10-40). Despite the malnutrition of the patient, plasma proteins were also in normal values.

3-months after commencing the treatment with GH and MT, plasma IGF-I values increased to $122.3 \mu \mathrm{g} / \mathrm{L}$ (normal). Plasma liver transaminases were still slightly increased, but this 
was no longer observed in the subsequent analysis performed during the 16-months of treatment. Plasma IGF-I remained in normal values during the total period of treatment ranging between 125 and $141 \mu \mathrm{g} / \mathrm{L}$, while plasma IGFBP3 values oscillated between 3.1 and $4.2 \mu \mathrm{g} / \mathrm{L}$.

Blood analysis performed throughout the treatment did not show any alteration in all the parameters analyzed.

Treatment with GH and MT did not cause any adverse side effects, and the child grew 9 $\mathrm{cm}$, although his height was still slightly below p3.

\subsection{Genetic studies}

A first cytogenetic study carried out at age 1-year and 11-months (CHG-Array 60K, KaryoNIM) showed a deletion of 168 Kilobases in the cytoband 14q11.2, genomic coordinates 20.253.739-20.421.677. However this deletion was considered benign, since it had been described in the general population [19] and it had been not associated with any phenotypic effect on the carrier.

A further study, carried out at the age of 4-years and 2-months, just before admission in our Center, specifically designed for the patient (CHG-array containing 60,000 probes, from which 37,600 were composed by exonic sequences belonging to 453 genes involved in neurodevelopment; 16,900 probes uniformly distributed throughout the genome and 5,500 control probes) did not reveal any change in the gene dose that could relate to the pathology of the patient.

In spite of all this, and given the changes experienced by the patient after being treated, a new genetic study was carried out in his Hospital of reference, at the age of 6 years and 6months, that is almost 1-year after he was discharged from our Medical Center. In this case, the exoma was sequenced by using the Ion AmpliSeq ${ }^{\mathrm{TM}}$ Exome Kit for detecting variants in the sequence of a group of genes related to neurodevelopment. The results obtained detected the existence of a single nucleotide mutation in the gene IFIH1. Genomic coordinate was Chr2:162,273,932, nucleotide change: $c .2317 G>C$; this led to a change in glutamic acid 773 to glutamine whose result is the transcription of a pathologic protein (transcript NM_022168). The change, confirmed by conventional Sanger sequencing, was not observed in the parents of the patient, indicating that it was a de novo heterocygous mutation.

\section{Discussion}

This study describes the neurodevelopmental changes observed in a child initially diagnosed as diplegic spastic cerebral palsy, after being treated during 16-months with GH, MT and neurorehabilitation. The interest of this report is that the first genetic study carried out in the patient indicated the existence of a microdeletion 14q11.2, encompassing 4 genes, considered to be benign [19] and not responsible for the pathology observed in the patient. However, more recently, Zahir et al. described submicroscopic chromosome 14q11.2 deletions, ranging in size from $101 \mathrm{~kb}$ to $1.6 \mathrm{Mb}$, in three children that showed developmental delay and cognitive affectations, together with dysmorphic features [20]. They concluded that this chromosomic region has few stability, therefore de novo mutations or deletions may be relatively frequents in this region. We can not know whether the microdeletion first detected in our patient was responsible of his neurodevelopmental abnormalities, but some of the clinical characteristics described for the chromosome 14q11-q22 deletion syndrome (OMIM \# 613457) coincided with 
those found in our case [21]. For instance: failure to thrive and poor growth, microcephaly, poor head control, low-set ears, poor eye contact, neonatal hypotonia, delayed psychomotor development, inability to walk, lack of speech, hypertonia, spasticity, hyperreflexia, abnormal myelination. Contradictorily, a posterior genetic study, performed two years later, did not find any significant change in the gene dose that could justify the pathology of the patient. We don't know the reason for these contradictions between both studies, performed in different laboratories. Moreover, brain imaging studies also showed conflicting results because while the CT-SCAN performed at 2-years of age showed frontal bilateral calcifications, these were not observed in posterior magnetical resonance (MRI) imaging studies, which, in turn reported the existence of diffuse hypomyelination.

For these reasons, the patient had a diagnosis of cerebral palsy of unknown etiology when he came to our Medical Center for being treated. We treated him according to our protocols for children with cerebral palsy, both in terms of medication received (GH and MT) and neurorehabilitation $[22,23]$. GH was prescribed not only because of the low height of the patient and low plasma IGF-I values, a very common finding in children with cerebral palsy [24], but also for the known effects of the hormone on brain repair after a damage [25-28], particularly on cognition, executive functions and working memory [27, 29-32]. These GH effects were also observed in the patient, as the great improvement in the BDIST showed (14 months in 16 months of treatment), mainly in areas related to cognitive functions. This was specially important since, as described, the patient had suffered a very important regression in his neurodevelopmental milestones when he was 16-months old. In fact, some of his clinical manifestations had led to think of the existence of an autistic spectrum disorder. In turn, MT was given, preventively, because of its potent scavenging effects on the toxic reactive oxygen species (ROS) [33, 34], to which the brain is particularly sensitive, most likely elevated because of the clinical situation of the patient and the work carried out in his neurorehabilitation. Moreover, the clinical history indicated that neopterin, a marker of inflammatory-immune processes [35], had been detected to be elevated in a CSF sample of the patient; therefore, given the anti-inflammatory effects of MT $[36,37]$, we thought that the administration of this indolamine could add more beneficial effects to the patient.

The evolution of the patient has to be considered very good, mainly if we look at the starting point (chronological age: 4-years and 3-months; equivalent age: 10-months) after the regression he had suffered at an age in which brain development still continues, and since improvements continued after he was discharged, despite his motor limitations due to shortenig of tendons in lower limbs (abductors and Achilles).

Surprisingly, a new cytogenetic study carried out almost 1-year after discharge detected the existence of a mutation in the exon 12 of the gene IFIH1 (Interferon-induced helicase C domain-containing protein 1). This gene encodes the expression of a receptor (Melanoma Differentiation-Associated protein 5 [MDA5]), which acts as a cytoplasmic sensor of viral nucleic acids responding to them by activating a cascade of antiviral pathways; for instance inducing the expression of type I interferons (alpha and beta) and proinflammatory cytokines [38], therefore this gene plays a key role in the innate immune response. This physiological response becomes pathological when there is a mutation in the gene IFIH1 that leads to an overexpression of interferon 1, which is known as "interferon signature" [39]. Type 1 interferons regulate the 
transcription of a number of genes belonging to the immune system, by multiple and different signaling pathways (JAK/STATS, MAPK p38, Pi3K/Akt) [40]. Therefore it is logic that the existence of interferon signature has been linked to the development of a number of autoinmune diseases, such as systemic lupus erythematosus, Sjögren's syndrome, myositis, psoriasis, systemic sclerosis, rheumatoid arthritis and even it has been considered as a risk factor for developing type I diabetes in children with a genetic predisposition for this disease [41-43].

As described in the Introduction, interferon 1 (IFN- $\alpha$ ) overproduction was early related to the development of AGS [3, 4, 11, 12]. Since then, mutations in several genes leading to the apparition of interferon signature have been implicated in this syndrome [13-17, 44]; however it was not until 2014 that mutations in the IFIH1 gene were involved in the etiopathogeny of AGS $[45,46]$, demonstrating that IFIH1 mutations led to an increase in the production of type 1 interferon and to an increased transription of genes stimulated by this cytokine. Interestingly, in the last of these studies it was shown that all the patients with IFIH1 mutations presented autoantibodies, which suggested that these mutations contributed to the presentation of autoinmune phenotypes [46]. This was also observed in another study in patients presenting AGS [47]. INF-1 can be produced in the CNS, but also it can easily reach the CNS from plasma, modulating the function of microglia, astrocytes, neurons and oligodendrocytes; therefore, its overproduction leads to severe inflammatory encephalopathies, including AGS [48].

To our knowledge the mutation detected in the IFIH1 gene in our patient has never been previously described or has been associated with the development of AGS, but the geneticists who did the study identified it as pathological and associated with the occurrence of this syndrome. Moreover, since the diagnosis of AGS was made almost 1-year after discharge from our Medical Center, we did not measure CSF levels of IFN-1. Therefore, we don't know whether this cytokine is increased or its level of penetrance. However, the clinic of the patient was similar to that of two AGS cases previously described by the group of Rice [45], in which the onset of neurological regression with loss of motor and cognitive skills and onset of spasticity and dystonia appeared at 15-months of age. In our patient, the absence of some major clinical features usually found in AGS produced by IFIH1 gene mutations, such as clear intracranial calcifications, or deep white mater affectations, recurrent non-infectious fevers, chilblains, neonatal thrombocytopenia, among others, corresponds to the wide spectrum of the presentation of AGS $[49,50]$. Although the existence of AGS seemed to be clear in the case here described, we can not discard that some of the clinical features that the patient presented from birth were due to the microdeletion in cytoband 14q11.2 detected in the first genetic study carried out, as shown in OMIM database [21]. Then, the possibility exists that two genetic abnormalities contributed to the clinical features observed.

AGS is the consequence of inadequate immune activation, showing features of autoinflammatory and autoimmune disorders. These are the reasons by which different treatments combining corticoids with immunosuppressants or immunoglobulins have been used without known effectivity [51]. On the other hand, and given that the type 1 interferon-signature is a constant in AGS, independently of the causal genotype, other therapeutic approaches consider to block IFN-1 or its receptor by using monoclonal antibodies. More recently, the use of reverse transcriptase inhibitors, such as those used to treat HIV-1 infections, in children and adults has been suggested for treating AGS [for a detailed review, see ref. 51]. 
Therefore, how to explain the significant improvements achieved in our patient when the treatment with GH and MT began together with neurorehabilitation, after almost 3-years receiving intense rehabilitation without significant results?

Apart of the high number of beneficial effects that MT plays in the human organism, this hormone has anti-inflammatory properties and it is a mitochondrial protector [36, 37, 52]; in addition, it has been shown that, in rats, melatonin protects the liver against ischemia/reperfusion injury by inducing heme-oxygenase- 1 overexpression, which, in turn, suprresses the type 1 IFN signaling pathway dowstream of Toll-like receptor 4 [53], a transmembrane receptor whose activation leads to the induction of a type 1 IFN response in Trex 1 null mice [54], one of the genes whose mutations lead to AGS [44].

Since CSF or plasma levels of IFN-1 were not measured in our patient either before or during treatment with MT we can not affirm that the improvement obtained has been due to the blockade of IFN-1, but a recent study utilizing a novel MT derivative which possessess stronger anti-inflammatory properties than the physiological hormone, demonstrated that its potent effects are exerted via the regulation of IFN-dependent signaling patway from Toll-like receptors [55]. Moreover, we treated a women with rheumatoid arthritis, an autoimmune disease with interferon-signature, with $100 \mathrm{mg} /$ day of MT and one month after beginning the treatment her joint pains had disappeared and plasma markers of inflammation were fully normalised, something that had never happened during the long time that she had been treated with immunosuppressants or corticoids (unpublished data).

In summary, we believe that the positive results obained in our AGS patient have been produced by the effects of MT on IFN-1 and inflammation, then allowing GH and neurorehabilitation to act on his psychomotor disabilities and growth.

Currently the dose of MT has been increased to $100 \mathrm{mg} /$ ay and his cognitive and motor skills continue to being improved.

\section{Materials and Methods}

The patient was a 4-years and 3-months old male born by scheduled caesarean carried out at week 40, due to loss of fetal growth and failure of labor induction. He was the unique child from a nonconsanguineal marriage. His mother had suffered two previous abortions in the first trimester of pregnancy. The mother did not have any kind of toxic habits; she did not take any kind of pharmaceutical drugs during their pregnancies, nor she had been exposed to toxic agents or had suffered from an illness.

As the only significant family history, the child had a cousin with Asperger's syndrome. Apgar score at birth was 9/10 (1 min/5 min); his weight at birth was $2.740 \mathrm{~kg}(<\mathrm{p} 3)$ and his size was $46 \mathrm{~cm} \quad(<\mathrm{p} 3)$. The child presented microcephaly (head circumference: $34 \mathrm{~cm}$ ). Gastroesophageal reflux existed until 14-months of age. The evolution of weight and growth were very low $(\ll<$ p3) from birth until 16-months of age where there was a stagnation; however, at 9months old he was able to initiate sedestation, and at 16-months of age began ambulation, but both were totally lost in the next six months. At this time axial hypotony and spasticity in lower limbs appeared, while in upper limbs distony existed, more marked in the right side. At 3-years old he was only able to say two words. Therefore he began to communicate through his shown to be elevated own sign language. 
Blood analysis were normal (hematimetry, biochemistry and hormones), with the exception of increased plasma IgE values $(622 \mathrm{UI} / \mathrm{mL}$, normal: $<40 \mathrm{UI} / \mathrm{mL})$, which was attributed to intolerance to cow's milk, and persistently low values of plasma IGF-I $(<25 \mu \mathrm{g} / \mathrm{L})$. All types of metabolic (including organic acids and very long chain fatty acids) and serological studies (Borrelia burgdoferi, Cytomegalovirus and Herpes simple) were normal. Interestingly, an increased value of neopterin in CSF was observed (198 nmol/L; normal: $12-55 \mathrm{nmol} / \mathrm{L}$ ), but it was thought to be the expression of an inflammatory process or an autoinmune disease.

A first genetic study (CHG-Array 60K, KaryoNIM) showed a deletion of 168 Kilobases in the cytoband 14q11.2. However this deletion was considered benign [19]. A new genetic study, performed 3-years later showed no loss in the genetic endowment, therefore contradicting the previous genetic findings.

A CT-SCAN performed at 2-years old indicated the existence of bilateral frontal subcortical hyperdensic foci, secondary to calcifications. However, these were not observed in further cerebral MRIs, which, in turn, showed moderated diffuse hypomyelination for the age of the patient. A MRI study of the spinal cord indicated that there were no anomalies in this territory.

Electroencephalograms were normal. Electromyograms showed that motor and sensitive conductions were normal in the lower limbs. Auditory and visual evoked potentials were normal.

Based on these data the patient was diagnosed of cerebral palsy of unknown etiology, mainly manifested by spastic paraplegia and anarthria.

Since the clinical symptoms began to be manifested the child received an intense rehabilitation (Doman's method) without significant improvements.

At admission to our Medical Center (age 4 years and 3 months), the patient had a height $(85 \mathrm{~cm})$ strongly below p3 for his age, Similarly, his body weight $(9,200 \mathrm{~kg})$ was clearly below p3. The patient presented microcephaly, low-set ears, enognatism, anarthria and lingual hypotonia, axial hypotony with poor cephalic control and unstable sedestation. There was spasticity in the lower limbs (MAS grade 3), presenting an extension pattern. Spasticity had led to a marked shortening of the abductors and Achilles tendon; therefore, there was equine feet and scissoring gait when the patient tried to walk with the support of his parents. The angle of abduction had a severe limitation, Babinski sign was + and marked hyperreflexia of the lower limbs existed. Interestingly, the patient showed a strong hypersensitivity to any stimuli in hands and feet, as well as refusal to be fed and only was able to eat crushed food. Vomiting reflex was advanced. No nistagmus existed and there was poor eye contact. However, according to the information given by his parents, the child liked to watch cartoons in TV. The patient never suffered seizures, and there were some small stereotypies.

Once the medical examination was performed, standard tests for evaluating childhood developmental milestones (GMFM-88, BDIST and the WeeFim test) were carried out. These tests were repeated at 3-months intervals.

Routine blood analysis (hematimetry and biochemistry) and plasma TSH, fT4, cortisol and IGF-I were performed before commencing medical and neurorehabilitation treatments. All plasma values were normal, with the exception of IGF-I which was below the lower limit of 
normality for his age $(<25 \mu \mathrm{g} / \mathrm{L})$. These blood analysis were repeated every 3-months until discharge.

Medical treatments consisted in Growth Hormone (GH) and Melatonin (MT). GH (Nutropin, Ipsen) was given at a dose of $0.04 \mathrm{mg} / \mathrm{kg} /$ day (5 days/week) for 3-months, followed by 15 days resting. After this, the same schedule was performed during the 16-months that the patient was treated in our Center, adjusting the GH dose to the body weight every 3-months. MT was given orally before bedtime at an initial dose of $20 \mathrm{mg} /$ day. 3-months later the dose was increased to $50 \mathrm{mg} /$ day. These doses of MT were prepared as a syrup, because of the aversive behavior of the patient to be fed, by magistral formula.

Neurorehabilitation (45 minutes/session, 5 days/week) consisted in: physiotherapy, speech therapy, neuropsychology and occupational therapy.

Treatments were conducted according to the protocols of the Medical Center Foltra and in compliance with the Spanish legislation for using GH and MT "off label" and the Code of Ethics of the World Medical Association (Declaration of Helsinki). Signed informed consent for the use of GH and MT was obtained from the parents of the patient.

Videos were taped in the Medical Center Foltra. Images in this manuscript have been obtained from these videos, excepting the image in Figure 3 which was sent by the parents of the patient. The parents of the patient gave signed informed consent for the publication of the results and images, but due to ethical reasons we decided to blur his face for avoiding any recognition of him.

\section{Conclusions}

According to our results, a combination of a medical treatment with MT plus GH, together with neurorehabilitation, may be very useful for the treatment of children with AGS. The treatment is safe and its utility has to be greater the earlier one begins once the disease is detected.

\section{Acknowledgements}

We acknowledge the parents of the patient for giving us written informed consent to publish this case report and any accompanying images.

Funds for covering the costs to publish in open access proceeded from Foundation Foltra (Teo, Spain).

\section{Author Contributions}

Jesús Devesa and Carlos I. Puell conceived and designed the work to be carried out and the medical treatment given; Alba Alonso, Patricia Porto, Ana Quintana and María Carrillo performed the work carried out in neurorehabilitation and successive control tests; Jesús Devesa and Carlos I. Puell analysed the data; Pablo Devesa looked for genetic references and clinical symptoms in databases; Jesús Devesa wrote the manuscript. All the authors have read and approved the final version of the manuscript.

Radiological, MRI and genetic studies were performed in the Hospital Universitario La Fe (Valencia, Spain), with the exception of the first genetic study (Clínica VistaHermosa, Alicante, 
Spain). X-ray studies of the hips were performed inn the Hospital La Rosaleda (Santiago de Compostela, Spain).

\section{Conflicts of Interest}

The authors declare no conflict of interest.

\section{References}

1. Aicardi, J.; Goutières, F. A progressive familial encephalopathy in infancy with calcifications of the basal ganglia and chronic cerebrospinal fluid lymphocytosis. Ann. Neurol. 1984, 15, 49-54.

2. Giroud, M.; Gouyon, J.B.; Chaumet, F.; et al. A case of progressive familial encephalopathy in infancy with calcification of the basal ganglia and chronic cerebrospinal fluid lymphocytosis. Childs Nerv. Syst. 1986, 2, 47-48.

3. Goutières, F.; Aicardi, J.; Barth, P.G.; Lebon, P. Aicardi-Goutières syndrome: an update and results of interferon-alpha studies. Ann. Neurol. 1998, 44, 900-907.

4. Lebon, P.; Badoual, J.; Ponsot, G.; Goutières, F.; Hemeury-Cukier, F.; Aicardi, J. Intrathecal synthesis of interferon-alpha in infants with progressive familial encephalopathy. J. Neurol. Sci. 1988, 84, 201-208.

5. Mehta, L.; Trounce, J.Q.; Moore, J.R.; Young, I.D. Familial calcification of the basal ganglia with cerebrospinal fluid pleocytosis. J. Med. Genet. 1986, 23, 157-160.

6. Diament, A.J.; Machado, L.R.; Cypel, S.; Ramos, J.L. [Syndrome of calcifications of basal ganglia, leukodystrophy and chronic lymphomonocytic pleocytosis of the cerebrospinal fluid: report of a case]. Arq. Neuropsiquiatr. 1986, 44, 185-190.

7. Bönnemann, C.G.; Meinecke, P. Encephalopathy of infancy with intracerebral calcification and chronic spinal fluid lymphocytosis-another case of the Aicardi-Goutières syndrome. Neuropediatrics. 1992, 23, 157-161.

8. Tolmie, J.L.; Shilito, P.; Hughes-Benzie, R.; Stephenson, J.B. The Arcadi-Goutières syndrome (familial, early onset encephalopathy with calcifications of the basal ganglia and chronic cerebrospinal fluid lymphocytosis). J. Med. Genet. 1995, 32, 881-884.

9. Verrips, A.; Hiel, J.A.; Gabreëis, F.J.; Wesseling, P.; Rotteveel, J.J. The Arcadi-Goutières syndrome: variable clinical expression in two siblings. Pediatr. Neurol. 1997, 16, 323-325.

10. Kumar, D.; Rittey, C.; Cameron, A.H.; Variend, S. Recognizable inherited syndrome of progressive central nervous system degeneration and generalized intracranial calcification with overlapping phenotype of the syndrome of Aicardi and Goutières. Am. J. Med. Genet. 1998, 75, 508-515.

11. McEntagart, M.; Kamel, H.; Lebon, P.; King, M.D. Arcadi-Goutières syndrome: an expanding phenotype. Neuropediatrics. 1998, 29, 163-167.

12. Ostergaard, J.R.; Christensen, T.; Nehen, A.M. A distinct difference in clinical expression of two siblings with Arcadi-Goutières syndrome. Neuropediatrics. 1999, 30, 38-41. 
13. Crow, Y.J.; Jackson, A.P.; Roberts, E. et al. Aicardi-Goutières Syndrome Displays Genetic Heterogeneity with One Locus (AGS1) on Chromosome 3p21. Am. J. Hum. Genet. 2000, 67, 213221.

14. Crow, Y.J.; Hayward, B.E.; Parmar, R. et al. Mutations in the gene encoding the $3^{\prime}-5^{\prime}$ DNA exonuclease TREX1 cause Aicardi-Goutières syndrome at the AGS1 locus. Nat. Genet. 2006, 38, 917-920.

15. Crow, Y.J.; Leitch, A.; Hayward, B.E. et al. Mutations in genes encoding ribonuclease H2 subunits cause Aicardi-Goutières syndrome and mimic congenital viral brain infection. Nat. Genet. 2006, 38, 910-916.

16. Rice, G.I.; Bond, J.; Asipu, A. et al. Mutations involved in Aicardi-Goutières syndrome implicate SAMHD1 as regulator of the innate immune response. Nat. Genet. 2009, 41, 829-832.

17. Rice, G.I.; Kasher, P.R.; Forte, G.M. et al. Mutations in ADAR1 cause Aicardi-Goutières syndrome associated with a type I interferon signature. Nat. Genet. 2012, 44, 1243-1248.

18. Hanna, S.E.; Bartlett. J.; Rivard, L.M.; Rusell, D.J. Reference Curves for the Gross Motor Function Measure: Percentiles for Clinical Description and Tracking Over Time among Children with Cerebral Palsy. Phys. Ther. 2008, 88, 596-607.

19. Iafrate, A.J.; Feuk, L.; Rivera, M.N. et al. Detection of large-scale variations in the human genome. Nat. Genet. 2004, 36, 949-951.

20. Zahir, F.; Firth, H.V.; Baross, A. et al. Novel deletions of 14q11.2 associated with developmental delay cognitive impairment and similar minor anomalies in three children. J. Med. Genet. 2007, 44, 556-561.

21. OMIM Org. http://www.omim.org/clinicalSynopsis/613457 (archived on 1862010 ).

22. Reimunde, P.; Rodicio, C.; López, N.; Alonso, A.; Devesa, P.; Devesa, J. Effects of recombinant growth hormone replacement and physical rehabilitation in recovery of gross motor function in children with cerebral palsy. Ther. Clin. Risk Manag. 2010, 6, 585-592.

23. Devesa, J.; Alonso, B.; Casteleiro, N. et al. Effects of recombinant growth hormone (GH) replacement and psychomotor and cognitive stimulation in the neurodevelopment of GHdeficient (GHD) children with cerebral palsy: a pilot study. Ther. Clin. Risk Manag. 2011, 7, 199206.

24. Devesa, J.; Casteleiro, N.; Rodicio, C.; López, N.; Reimunde, P. Growth hormone deficiency and cerebral palsy. Ther. Clin. Risk Manag. 2010, 6, 413-418.

25. Arce, V.M.; Devesa, P.; Devesa, J. Role of growth hormone (GH) in the treatment on neural diseases: from neuroprotection to neural repair. Neurosci. Res. 2013, 76, 179-186.

26. Devesa. J.; Díaz-Getino, G.; Rey, P. et al. Brain Recovery after a Plane Crash: Treatment with Growth Hormone (GH) and Neurorehabilitation: A Case Report. Int. J. Mol. Sci. 2015, 16, 30470-30482.

27. Devesa, J.; Lema, H.; Zas, E.; Munín, B.; Taboada, P.; Devesa, P. Learning and Memory Recoveries in a Young Girl Treated with Growth Hormone and Neurorehabilitation. J. Clin. Med. 2016, 5, pii: E14. 
28. Devesa, J.; Almengló, C.; Devesa, P. Multiple Effects of Growth Hormone in the Body: Is it Really the Hormone for Growth? Clin. Med. Insights Endocrinol. Diabetes. 2016, 9, 47-71.

29. Nyberg, F. The role of the somatotrophic axis in neuroprotection and neuroregeneration of the addictive brain. Int. Rev. Neurobiol. 2009, 88, 399-427.

30. Nyberg, F.; Hallberg, M. Growth hormone and cognitive function. Nat. Rev. Endocrinol. 2013, 9, 357-365.

31. Muresanu, D.F.; Sharma, A.; Lafuente, J.V. et al. Recombinant human growth hormone improves cognitive capacity in a patient exposed to chronic opioids. Acta Anaesthesiol. Scand. 2014, $58,759-765$.

32. Park, K.D.; Lim, O.K.; Yoo, C.J. et al. Voxel-based statistical analysis of brain metabolism in patients with growth hormone deficiency after traumatic brain injury. Brain Inj. 2016, 30, 407413.

33. Reiter, R.J. Functional aspects of the pineal hormone melatonin in combating cell and tissue damage induced by free radicals. Eur. J. Endocrinol. 1996, 134, 412-430.

34. Reiter, R.J. Antioxidant actions of melatonin. Adv. Pharmacol. 1997, 38, 103-117.

35. Molero-Luis, M.; Fernández-Ureña, S.; Jordán, I. et al. Cerebrospinal fluid neopterin analysis in neuropediatric patients: establishment of a new cut-off value for the identification of inflammatory-immune mediated processes. PLoS One. 2013, 8, e83237.

36. Sánchez, A.; Calpena, A.C.; Clares, B. Evaluating the Oxidative Stress in Inflammation. Int. J. Mol. Sci. 2015, 16, 16981-17004.

37. Dong, Y.; Fan, C.; Hu, W. et al. Melatonin attenuated early brain injury induced by subarachnoid hemorrhage via regulating NLRP3 inflammasome and apoptosis signaling. J. Pineal Res. 2016, 60, 253-262.

38. Kato, H.; Takeuchi, H.; Mikamo-Satoh, E. et al. Length-dependent recognition of doublestranded ribonucleic acids by retinoic acid-inducible gene-I and melanoma differentiationassociated gene 5. J. Exp. Med. 2008, 205, 1601-1610.

39. Baechler, E.C.; Batliwalla, F.M.; Karypis, G. et al. Interferon-inducible gene expression signature in peripheral blood cells of patients with severe lupus. Proc. Natl. Acad. Sci. USA. 2003, 100, 2610-2615.

40. Platanias, L.C. Mechanisms of type-I and type-II-interferon-mediated signalling. Nat. Rev. Immunol. 2005, 5, 375-386.

41. Hall, J.C.; Rosen, A. Type I interferons: crucial participants in disease amplification in autoimmunity. Nat. Rev. Rheumatol. 2010, 6, 40-49.

42. Di Domizio, J.; Cao, W. Fueling Autoimmunity: Type I Interferon in Autoimmune diseases. Expert. Rev. Clin. Immunol. 2013, 9, 10.1586/eci.12.106.

43. Ferreira, R.C.; Guo, H.; Coulson, R.M.R. et al. A Type I Interferon Transcriptional Signature Precedes Autoimmunity in Children Genetically at Risk for Type 1 Diabetes. Diabetes. 2014, 63, 2538-2550. 
44. Rice, G.I.; Forte, G.M.; Szynkiewicz, M. et al. Assessment of interferon-related biomarkers in Aicardi-Goutières syndrome associated with mutations in TREX1, RNASEH2A, RNASEH2B, RNASH2C, SAMHD1, and ADAR: a case control study. Lancet Neurol. 2013, 12, 1159-1169.

45. Rice, G.I.; del Toro Duany, Y.; Jenkinson, E.M. et al. Gain-of function mutations in IFIH1 cause a spectrum of human disease phenotypes associated with upregulated type I interferon signaling. Nat. Genet. 2014, 46, 503-509.

46. Oda, H.; Nakagawa, K.; Abe, J. et al. Aicardi-Goutières Syndrome Is Caused by IFIH1 Mutations. Am. J. Hum. Genet. 2014, 95, 121-125.

47. Lee-Kirsch, M.A.; Wolf, C.; Günther, C. Aicardi-Goutières syndrome: a model disease for systemic autoimmunity. Clin. Exp. Immunol. 2014, 175, 17-24.

48. Blank, T.; Prinz, M. Type I interferon pathway in CNS homeostasis and neurological diseases. Glia. 2017, 65, 1397-1406.

49. Crow, Y.J.; Manel, N. Aicardi-Goutières syndrome and the type I interferonopathies. Nat. Rev. Immunol. 2015, 15, 429-440.

50. Svingen, L.; Goheen, M.; Godfrey, R. et al. Late diagnosis and atypical brain imaging of Aicardi-Goutières syndrome: are we failing to diagnose Aicardi-Goutières syndrome-2? Dev. Med. Child Neurol. 2017, Aug 1. doi: 10.1111/dmcn.13509. [Epub ahead of print].

51. Crow, Y.J.; Vanderver, A.; Orcesi, S.; Kuijpers, T.W.; Rice, G.I. Therapies in AicardiGoutières syndrome. Clin. Exp. Immunol. 2014, 175, 1-8.

52. Cardinali, D.P.; Vigo, D.E. Melatonin, mitochondria, and the metabolic syndrome. Cell Mol. Sci. 2017, Aug 17. doi: 10.1007/s00018-017-2611-0. [Epub ahead of print]

53. Kang, J.W.; Lee, S.M. Melatonin inhibits type 1 interferon signaling of toll-like receptor 4 via heme-oxygenase-1 induction in hepatic ischemia/reperfusion. J. Pineal Res. 2012, 53, 67-76.

54. Stetson, D.B.; Ko, J.S.; Heidmann, T.; Medzhitov, R. Trex1 prevents cell-intrinsic initiation of autoimmunity. Cell. 2008, 134, 587-598.

55. Shim, D.W.; Shin, H.J.; Han, J.W. et al. A novel synthetic derivative of melatonin, 5hydroxy-2'-isobutyl-streptochlorin (HIS) inhibits inflammatory responses via regulation of TRIFdependent signaling and inflammasome activation. Toxicol. Appl. Pharmacol. 2015, 284, 227-235. 